\title{
OCTAVA MESA REDONDA
}

\section{Pelvimetría}

1. Al doctor Agüero: -En los casos de aplicación de fórceps, ¿siempre los aplican medios? ¿Qué morbilidad materna e infantil han tenido en estos casos?

2. Al doctor Agüero: -Morbilidad materna y fetal en aplicación de fórceps en estrecheces del estrecho medio.

Doctor Agüero: - Los fórceps medios no siempre son aplicados por problemas pélvicos. En realidad la mayoría se deben a problemas de rotación de transversa o de posteriores. Nuestro índice de morbilidad y mortalidad materna e infantil fue muy pequeño. No hubo ninguna muerte materna por asunto de estrecheces pélvicas, y en cuanto a los niños la mortalidad prenatal fue de $0,9 \%$. Entre esos casos hubo muertes de fetos, no achacables al procedimiento, como defectos congénitos, hidrocefalia, etc., es decir, de fetos muertos antes de la prueba de trabajo. En un estudio que hicimos de ochocientos fórceps indicados en nuestra clientela privada, la incidencia nuestra de mortalidad en los fórceps medios fue de $0,9 \%$. Solamente hubo unos dos o tres casos de niños lesionados en la operación, fueron lesiones muy leves de mejilla y del aparato auricular. Si tene- mos un porcentaje de mortabilidad materna evidenciable especialmente en desgarros de partes blandas, cuello, vagina y periné, no achacables a la operación misma. Tenemos tres casos de lesiones del aparato urológico entre los ochocientos casos de fórceps medios indicados, incluyendo lesiones de vejiga y de uretra.

Doctor Agüero: - Quiero hacer una breve alusión a la anestesia en la aplicación del fórceps. En estos casos estimamos que el mejor pronóstico para el feto, la madre y la comodidad del operador, se da por medio de anestesias de bloqueo ya sea epidural o subdural.

3. Al profesor José del Carmen Acosta: - ¿Qué concepto tiene sobre la Sinfisiotomía?

Doctor Acosta:-Como observamos en los relatos hechos especialmente por los representantes argentinos, la Sinfisiotomía en la Argentina misma, en donde tuvo gran auge, después de los trabajos de Zárate, que la revivió a la antigua Sinfisiotomía, que ya estaba prácticamente abandonada, hoy día ha ido disminuyendo y vemos que según el último relato sólo el uno por ciento de los casos intervenidos figura. Encre nosotros, desde la época de los traba- 
jos de Zárate, se hizo un estudio e inclusive se hicieron tesis sobre la aplicación y técnica de la Sinfisiotomía. En algunos casos dio resultados buenos, pero también ocurrieron accidentes muy molestos de lesiones vesicales, los menos, pero lesiones del plejo retropúbico con hemorragia, y en algunos casos molestias prolongadas debidas a dificultades en la marcha por movilidad persistente en la sínfisis. Por esa razón la Sinfisiotomía está prácticamente abandonada desde hace muchos años entre nosotros. En los casos extremos de distocia por desproporción se recurre a la cesárea, y en los que hay compatibilidad se recurre a la aplicación de fórceps.

Doctor Martini: - No me sorprende, puesto que para muchos la Sinfisiotomía es anacrónica. Realmente tiene un bien justificado desprestigio, por los desastres que ocurrieron. Pero dichos desastres se debieron no a la Sinfisiotomía en sí, sino a los abusos y defectos en la técnica de los operadores. Yo tengo una escuela, digamos descendiente de Zárate, y puedo hablar de ella con conocimiento de causa. Es tan importante en ella que el parto termine espontáneamente, como que los ayudantes sepan exactamente lo que están haciendo. Generalmente se opina de la Sinfisiotomía sin conocerla porque no se practica. La Sinfisiotomía con fórceps es un gran riesgo, es necesaria una buena dinámica, que los actuales métodos de preparación para el parto favorecen. Otra cosa importante es el exacto diagnóstico del grado de proporción. Considero de acuerdo a la experiencia que tengo, a los recursos que existen actualmente para conducir el parto, a la forma inteligente de utilizar las estimulaciones fisiológicas de la dinámica, que no tiene los riesgos que se le atribuyen. La ventosa obstétrica de Malmström permite, en aquellos casos en que por error de indicación respecto de la actividad contráctil, colocar sin los riesgos de los fórceps, una cabeza que está alta y que no descendería por falta de proporción.

Doctor Agüero: —En Caracas se abandonó en el 45 después de resultados desastrosos, aun cuando era practicada por nuestros maestros, gente de experiencia, y no creo que tenga cabida la Sinfisiotomía dentro de la medicina actual. Yo no tengo más experiencia, que la de haber asistido como "inteligente" a una operación. El doctor Castelazo me pide que diga que él tampoco tiene experiencia al respecto.

4. Doctor Castelazo: - ¿Cuál es la medida del diámetro ileotrocantéreo y emplea usted la radiopelvimetría para este estudio?

5. - ¿Radiocéfalo-pelvimetría debe hacerse en pacientes primigestantes seleccionadas clínicamente $\mathrm{o}$ indiscriminadamente?

Doctor Castelazo: - Nosotros no utilizamos en nuestra práctica mediciones de pelvimetría externa, más que en 15 que se refiere al estrecho inferior, porque consideramos que es una información muy indirecta de la real capacidad de la pelvis obstétrica y que en la actualidad hay otros medios para hacerlo. Las dimensiones son menores, entre 29 y $30 \mathrm{cms}$. 
En lo que se refiere a la otra pregunta, me parece muy particularmente interesante, porque se trata de un asunto que tiene actualidad y acerca del cual hay divergencias de opinión entre diversas autoridades actuales de la materia. Nosotros no consideramos en lo personal que sea lo mejor el practicar la pelvicefalometría en todas las primigestas en forma general. No es por temor al efecto nocivo de las radiaciones, ni por no exponer inútilmente a las pacientes o a los fetos a este efecto; no practicamos la pelvicefalometría radiológica en todas las primigestas porque lo consideramos innecesario. Evidentemente que si a las treinta y siete o treinta y ocho semanas del embarazo en una primigesta, encontramos que la cabeza está ya metida en la pelvis, y la medición clínica del estrecho inferior nos muestra una capacidad y una morfología normales, nos parece un poco ocioso hacer una pelvicefalometría radiológica; entonces recurrimos a este procedimiento cuando la clínica nos permite algún dato que oriente hacia alguna alteración de la morfología o dimensiones pelvianas, que pueda interftrir de una manera fisiológica con el parto por vías naturales. Sí considero que tiene utilidad estadística el practicar pelvicefalometría sistemática a las primigestas, pero en la práctica clínica lo considero innecesario.

Doctor Martini: - Sobre la pelvimetría externa estoy de acuerdo con el doctor Castelazo; sin embargo, nosotros mantenemos una especie de gimnasia śrniológica, con fines didácticos o docentes, y además comprobamos cierto dolor presuntivo de las anomalías cuan- do éstas ocurren en los resultados de este examen.

Doctor Caravías: - A la pregunta primera, se me ocurre que debe ser el bitrocantéreo si el diámetro medio es de $31 \mathrm{cms}$.

Referente a la otra pregunta, debo responder que por razones fundamentales había que hacer el estudio, no sólo en las pelvis enfermas, sino también el estudio radiológico pelvimétrico. A tal efecto utilizamos el enorme material que tiene el Instituto de Radiología y Fisioterapia de Buenos Aires.

Al doctor Acosta: - ¿Qué concepto tiene de la pelvis relativamente estrecha y conducta durante el parto?

Doctor Acosta Bendek: - Cuando uno encuentra un diámetro promontoretropúbico menor de $10 \mathrm{cms}$. o un conjugado diagonal menor de $11 \mathrm{cms}$. y con un diámetro transverso menor de $11 \mathrm{cms}$. Y en el estrecho medio, cuando encontramos un diámetro biespinoso menor de $10 \mathrm{cms}$. o un diámetro biisquiático menor de 8 o $7 \mathrm{cms}$. Si encontramos esos diámetros en una pelvis, podemos considerarnos frente a un caso de estenosis relativa. Con relación a la conducta, yo espero siempre la prueba del trabajo de parto, y entonces tenemos que analizar varios factores: el tamaño del niño, que es sumamente importante; cómo se desarrolla ese trabajo de parto; la actividad contráctil del útero; después de ruptura de membranas de tres cms. de dilatación, cómo sigue esa dilatación; si ese niño queda estacionado o si sigue un descenso normal. De tal manera que con una apreciación muy minuciosa de 
ese trabajo de parto podemos nosotros decidir si el parto se puede hacer por las vías naturales o hay que interrumpirlo.

Profesor Acosta: — ¿Toda cesárea debe ir seguida para su nuevo parto de cesárea? ¿Es conveniente en una cesárea repetida dejar que comience el trabajo para practicarla?

Doctor Acosta: - Hay que hacer una diferenciación: si se trata una mujer con pelvis normal que haya requerido una intervención por una afección de orden obstétrico o una afección corriente ocurrida durante el parto; en estos casos no es necesario que en los partos posteriores la mujer sea sometida a cesáreas. Esto tiene validez, especialmente hoy día en que la cesárea corporal ha sido completamente abandonada en todos los medios obstétricos, y se recurre a la operación segmentaria, bien sea con incisión vertical o transversa del segmento, operaciones que dan mucho mayor seguridad en cuanto a la resistencia de la cicatriz y que no exponen a la mujer que ha sufrido dicha intervención a sufrirlas en los embarazos siguientes. Personalmentc lo he experimentado mucho, tanto en intervenciones que practiqué yo mismo, como por otros; y sin embargo, atendí partos posteriores a dicha intervención que se desarrollaron normalmente y por vías naturales. Esto en mujeres que habían tenido una cesárea por motivos accidentales. De manera que es raro que se tenga que practicar una cesárea en el siguiente parto con este tipo de pacientes. Es diferente cuando se trata de una desproporción céfalo-pélvica; en estos casos claro que es necesario evaluar el volumen del feto en el embarazo de que se esté tratando. Porque si la desproporción fue ocasionada por un exceso de volumen en un embarazo anterior y en el actual viene un feto de tamaño normal, o pequeño, tampoco requeriría la intervención; pero si subsiste la desproporción, es obvio que hay que repetir la intervención.

En lo que se refiere a la segunda pregunta, en los relatos que aquí se hicieron esta mañana se vio claramente que la mayoría son partidarios de que se deje comenzar el trabajo, pues eso facilita la intervención en la técnica misma, despliega el segmento inferior, lo amplía, facilita su incisión, especialmente tratándose de incisiones transversas; debemos pues aceptar como línea de conducta en la cesárea imperativa el esperar a que se inicie el trabajo, observando naturalmente a la mujer para darse cuenta de la extensión del segmento para intervenir en el momento oportuno, no esperar a que él se rompa.

Doctor Castelazo: - Estoy de acuerdo con el doctor Acosta en todos sus conceptos; simplemente quisiera insistir en un punto que me parece de mucha importancia y es la evaluación, en muchas ocasiones liviana, que se hace de la desproporción céfalo-pélvica, como indicación para una operación cesárea. Es bastante frecuente, y esto no es un fenómeno observado únicamente en Latinoamérica, es bastante frecuente que el médico que vigila el trabajo del parto, al observar que pasa el tiempo y el trabajo no progresa satisfactoriamente, 
no se detiene a hacer una evaluación minuciosa de las razones por las que el parto está desarrollándose mal, y con la etiqueta de desproporción opera a la paciente. Estas cifras que hemos presentado al final de nuestro relato muestran que entre el 20 y el $60 \%$ de pacientes que fueron operadas por desproporción, en embarazos subsiguientes dan a luz, por vías naturales, fetos completamente a término. Cabría invocar que el feto del embarazo anterior fue más chico, o que hubo una posición menos favorable, una actitud menos favorable de la cabeza en el embarazo anterior. Pero esto nos llevaría a que existieran cifras de pacientes que dan a luz por la vía vaginal cuando hay desproporción, pero no tantas. Es una invitación para hacer destacar que la indicación desproporción debe ser en general mejor evaluada de lo que lo está siendo actualmente.

Doctor Agüero: - Cuando se presenta una estrechez pélvica hacemos la cesárea electiva sin esperar a que se inicie el trabajo. Cuando por el contrario es, como lo ha dicho Castelazo, por desproporción con pelvis normal, radiológicamente comprobada, entonces permitimos la prueba de trabajo. En efecto hay un porcentaje muy elevado de partos espontáneos en estas mujeres; en el momento es de un $30 \%$ de partos espontáneos en mujeres operadas por desproporción en el primer embarazo. Con respecto al momento para efectuar la operación, en el primer caso, de estrechez pélvica, hacemos la operación electivamente sin esperar el trabajo del parto, unos seis u ocho días antes de la fecha probable del parto. Esto por no hacer correr a la enferma el riesgo de una desunión uterina.

Doctor Avendaño: - De acuerdo con el tema que estamos tratando, de si toda cesárea debe ir seguida en embarazos posteriores de nuevas cesáreas, tratándose de primera cesárea por vicio pelviano. A este respecto, nosotros hemos evolucionado haciéndonos más radicales en la tendencia operatoria. También hemos establecido una clasificación adecuada a este criterio; es así como lo mencionamos en el correlato, que consideramos una pelvis relativamente estrecha, una pelvis moderadamente estrecha y la absolutamente estrecha. Solamente en el primer grado admitiríamos una prueba de trabajo para una oportunidad; pero, como decíamos, somos en esto cada vez más desconfiados de los riesgos de la dehiscencia, riesgos que si bien es cierto pueden salvarse frente a la madre, representan gravísimos peligros para el feto. En cuanto a la conveniencia de practicar la segunda intervención de la cesárea iterativa después de algún tiempo, o practicarla de tipo electivo, me pronuncio francamente hacia la de tipo electivo, es decir, a practicarla cuanto antes, si ésta está indicada. A este respecto hemos introducido una innovación en la evaluación del grado de pureza ovular, denominando estos casos grado cero. Surot comenzaba por el grado uno, nosotros ponemos grado 0 a esta cesárea en la cual el huevo está íntegro, aséptico, y en el cual le damos la máxima garantía a la paciente en cuanto a la evolución postoperatoria y en cuanto a la prevención de la dehiscencia. 
Doctor Martini: -Nosotros indicamos única y exclusivamente cesárea a priori en los casos de estrechez absoluta. En los casos de estrechez relativa el antecedente de cesárea no contraindica para nosotros la prueba del parto. Desde luego estamos de acuerdo con el doctor Castelazo en que muchas veces hacen diagnósticos de desproporción que no son tales diagnósticos. Eso significa que es necesario intensificar la preparación del médico obstetra antes de independizarlo para que resuelva el caso. Es muy frecuente en los servicios hospitalarios que los índices operatorios sean altos en determinadas épocas, cuando se inician nuevos equipos de guardia. Nosotros hemos reducido notablemente el índice operatorio de la vía alta tomando esta práctica: la indicación de la vía alta debe ser hecha siempre por lo menos por dos especialistas experimentados. Lo cual entonces está muy de acuerdo con lo que dice el doctor Castelazo. Muchas veces en nuestra especialidad se hacen indicaciones de drogas estimulantes o sedantes sin haberse hecho el diagnóstico preciso de la distocia.

-Experiencia de la Mesa sobre la aplicación de la maniobra del doctor Ramírez Merchán en las distocias pélvico-fetales no graves.

Doctor Agüero: —La maniobra del doctor Ramírez Merchán es una maniobra de rotación de la cabeza. Para eso se necesita hacer una evaluación clínica del tipo de pelvis, porque es sumamente importante conocer exactamente qué tipo de pelvis tenemos en frente. Ya que esta maniobra, como es obvio, da los mejores resultados cuando se trata de un tipo de pelvis ginecoide. Los fracasos que ha habido en esta maniobra se han debido al quererla aplicar a todo tipo de pelvis.

Para el doctor Martini: - ¿Qué técnica usan en Sinfisiotomía, Zárate o Palacios Costa-Falsía?

Doctor Martini: _El que hace la pregunta debe de estar poco informado. No existe técnica de Palacios CostaFalsía. El era discípulo de Zárate y usaba su técnica. Ahora en lo que ha habido pequeñas modificaciones es respecto del instrumental; el bisturí de Schwartz, etc.

Al doctor Martini: —¿Cuál es el diámetro promonto-púbico mínimo como indicación en primíparas y multíparas?

Doctor Martini: —Es una pregunta muy simple, no me interesa el diámetro, lo que me interesa es la relación cabeza - pelvis. El problema de la distocia por desproporción es funcional. Lo difícil de la Sinfisiotomía es que no se puede esquematizar. Es necesario establecer la relación entre el tamaño del móvil y la amplitud del canal, para saber si se practica o no la Sinfisiotomía.

Al doctor Martini: — ¿Qué complicaciones han encontrado, y qué ventajas sobre la cesárea?

Doctor Martini: —La ventaja sobre la cesárea es esta: Estamos observando, y esto es un fenómeno universal, el aumento de frecuencia de un accidente que parecía propio de una época pasada; me refiero a ruptura uterina. Ahora con el aumento de las cesáreas estamos 
observando otro tipo de rotura del que se observaba antes, la dehiscencia de la cicatriz, que comienza ya a preocuparnos. De ahí que pensamos que es necesario disminuír en lo posible la frecuencia de las cesáreas. La Sinfisiotomía puede ser un recurso útil en este sentido para aquellos que la usen bien.

Para el doctor Castelazo: - Valor absoluto o relativo de la radiocefalopelvimetría en las presentaciones de nalgas en primigestas.

Doctor Castelazo: -Considero que tampoco en las primigestas, cuando hay una presentación pélvica, puede preconizarse la práctica sistemática de la radiocefalopelvimetría, porque considero que hay un número importante de casos en los cuales el clínico experimentado puede hacer una evaluación adecuada de la pelvis y una estimación sujetiva de que ocurrirá el parto pélvico sin problemas. Sin embargo, creo que la incidencia con la cual debe practicarse la pelvicefalometría radiológica en primigestas con presentación pélvica debe ser mucho mayor que cuando la presentación es cefálica. El procedimiento es más imperfecto cuando se trata de presentaciones pélvicas, ya que no hay esa relación de vecindad entre la cabeza y la pelvis, que permite evitar los errores por la distorsión que sufren las radiaciones. No obstante, creo que es una ayuda suficientemente importante al pronóstico del parto, que debe practicarse en todos los casos en que la más mínima duda asalte la mente del clínico de que puede haber problema de compatibilidad dimensional cefalopélvica. A este respecto y por tratar- se de una contribución latinoamericana, me parece importante destacar el procedimiento que ha ideado el doctor Terán de Costa Rica y su escuela de la Maternidad Carit, porque es un procedimiento que, aunque es de deducción indirecta, le ha permitido una proporción de acertados pronósticos verdaderamente excepcional. En la actualidad la estamos empleando y hasta este momento consideramos que el método tiene mucho porvenir.

Doctor Agüero: - Yo me cuento entre los que utilizamos y preconizamos la pelvimetría radiológica de rutina en las primigestas y en multíparas con antecedentes obstétricos oscuros. Desgraciadamente comprendo que es una práctica reservada a los hospitales que pueden hacerlo, pero considero que es una ayuda sin la cual es prácticamente imposible desarrollar normalmente un parto podálico especialmente en primigestas, ya que es imposible lograr una rotación de cabeza fetal con los métodos actuales. El procedimiento de TeránValls, que mencionó Castelazo, no ha sido todavía evaluado en cifras importantes, como para permitir juzgar su valor. Creo que debería hacerse. De modo pues que considero que debe hacerse la radiopelvimetría de rutina en toda primigesta en podálica, para lograr un porcentaje de morbilidad y mortalidad prenatal mucho menor que el actual. La cifra corriente de hospitales públicos es muy alta. Oscila entre un 15 a $30 \%$. En buenas manos, con un control radiológico previo, esa mortalidad puede rebajarse en mi opinión a las cifras del parto en cefálica. 
Doctor Acosta B.: - Para los que no tenemos a veces los medios para poder hacer el diagnóstico radiológico y métodos especializados en las presentaciones de nalgas, ya sea en primigestantes o multíparas, quiero insistir sobre todo en las multíparas, pues olvidamos que en muchas multíparas se pueden presentar casos de desproporción cefalopélvica, en partos posteriores. Entonces también tiene su importancia en las multíparas el tener como rutina hacer una radiopelvimetría, para poder hacer una evaluación radiopelvimétrica como también la posición del feto, y así evitar muchos problemas.
Doctor Martini: -Nosotros no tenemos experiencia en estudios radiopélvicos porque nos faltan recursos en nuestro medio para realizarlos. Desde luego que el estudio del canal del parto adquiere un valor extraordinario en la presentación pelviana. Nosotros agotamos los recursos clínicos para hacer1o. La razón es muy simple: desde el momento en que no puede hacerse la prueba del parto, no se puede exponer uno a un problema de desproporción de cabeza última, cuya solución a veces puede ser nefasta para el feto. Me parece de todos modos muy acertado lo que han dicho mis preopinantes. 